\title{
Scaling Up Inductive Learning with Massive Parallelism
}

\author{
FOSTER JOHN PROVOST \\ foster@nynexsi.com \\ NYNEX Science and Technology, 400 Westchester Avenue, White Plains. NY 10604 \\ JOHN M. $\triangle$ RONIS \\ $\operatorname{aronis}(e)$ sitt edu
}

Intelligent Systems laboratory, Universing of Pillsburgh, Pittsburgh, PA 15260

Editor: Douglas H. Fisher

\begin{abstract}
Alsstract. Miachine learning programs need ro scale up to very large data sets for several reasons, including increasing accuracy and discovering infrequent special cases. Current inductive learners perform well with hundreds or thousands of training examples, but in some cases, up to a million or more examples may be necessary to learn important special cases with confidence. These tasks are infeasible for current learning programs running on sequential machines. We discuss the need for very large data sets and prior efforts to scale up machine learning methods. This discusion motivates a stategy that exploits the inherent parallelism present in many learring algorithms. WL describe a parallel implementation of one inductive learning program on the CM-2 Connection Machine, show that it scales up to millions of examples, and show that it uncovers special-case rules that sequential learning programs, running on smaller datasets, would miss. The parallel version of the learning program is preferable to the sequential version for example sets larger than about $10 \mathrm{~K}$ examples. When leaning from a public-health databasc consisting of 3.5 million examples the paratlel rule-tearning system uncovered a surprisiny relarionship that has les in conciderable follow up researcts.
\end{abstract}

Keywords: inductive learnirg, parallelism. small disjuncts

\section{Introduction: Why Scale Lp?}

Current inductive learning programs cannot practically be used with very large data sets (e.g., a million or more examples). Catlett estimates (1991b) that real-world learning tasks using one million data items will require months on a dedicated workstation. This paper outlines reasons why very large data sets are necessary and summarizes past efforts to scale up machine learning methods. We then present an effective way to scale up a standard rule learner using massive parallelism and an implementation on the CM-2 Connection Machine. In a public-health domain, this program discovered relationships that could not have been found on current sequential machines. One relationship has led to considerable follow-up research by our public-health collaborators.

There are important reasons why machine learning methods must scale up to very large data sets. Perhaps the most obvious reason is to maximize accuracy. In the most comprehensive work to date on scaling up machine learning, Catlett (1991a) amassed a collection of very large data sets. In every domain, halving the size of the training set produced a statistically significant decrease in accuracy.

In many cases, degradation in accuracy when learning from small samples stems from over-fitting, due to high dimensionality of the concept description language or due to the 
need to allow the program to learn rules known as small disjucts (Holte, Acker \& Porter 1989), which correspond to special cases of the concept. Because small disjuncts cover fow data itcms, learning programs have difficulty learning these rules with confidence. Unfortunately, in some domains special cases account for a large portion of the concept (Danyluk \& Provost, 1993). In such domains, high-accuracy learning depends on the ability to lean special cases wcll. Noise further complicates the problem, because with a small sample it is impossible to tell the difference between a special case and a spurious data point (Danyluk \& Provost, 1993; Weiss, 1995).

Classilication accuracy aside, small disjuncts are olten of most interest to scientists and business analysts, since they are precisely the rules that were unknown previously; analysts usually know the common cases. Consider machine learning as an aid to publichealth research. It may be the case that, in general, fapanese-Americans have a low infant mortality rate. An inductive learner trying to describe the class Low-Infant-Mortality might look at linked birth/infant death data and produce the rule Japanese-American $\rightarrow$ Low-Infant Mortally. Leaming such a rule with high confidence is not a problem if the rule represents a substantial portion of the data, but if the rule covers only a small percentage of the data, a sample set of several thousand examples will not contain enough instances to inter the rule with confidence, if at all. Thus, if the sample sct contains only a few Japanese-Americans-a situation that is likely since they form such a small percentage $(0.3 \%)$ of the births in the U.S. - a learner could not draw conclusions about them with any degree of certainty.

In sum, a sample must be large enough to contain cnough instances of cach special case from which to generalize a rule with confidence. Learning rules for still smaller subgroups of the Japanese-American population would require still larger samples. For cxample, Japanese-Americans who live on the Jast Coast make up only $0.03 \%$ of the U.S. births. In order to have 30 examples from which to generalize a rule, one would need approximately 100,000 examples. In practice, it is desirable to have many more than 30 examples from which 10 generalize, to reduce the probability that a rule looks good by chance due to the generation and testing of many alternative hypotheses. For our example of Japanese-Americans living on the Fast Coast, increasing the number of examples required to generalize a rule by an order of magnitude pushes the total number of examples required up to one million. It is important to note that, in principle, scaling up does not eliminate the problem of small disjuncts; for any data set there could be ever-smaller special cases that could not be learned with confidence.

It chould be clear that scaling up to very large data sets implies, in part, that faster learners must be developed. There are, of course, other motivations for very fast learners. For example interactive machine learning (Bunine, 1991), in which a machine learnet and a human analysi interact in real time, requires very last learning algorithms in order to be practical. Nutomatic bias selection (Gordon \& desJardins, 1995) also requires very fast learners, because such systems evaluate learning on multiple biases; cach evaluation may involve mulfipts rune to produce performance statistics (e.g. with cross validation), and experimenting with many hiases also requires large data sets to avoid over fitting due to bias selection. In addition to implications for learning time, scaling up to very large data sote may require space efticient algorithme for spate-limited platforms. 
We now turn our attention to a number of strategies that scek to scale up learning methods to very large data sets and/or that have been designed with the related goals of reducing learning time or space complexity.

\section{Scaling Up Inductive Learning: What has heen done?}

There are several approaches one might take to apply symbolic machine learning to very large problems. A straightforward, alheit limited, strategy for scaling up is to use a very fast, but simple, method. This strategy may seem silly until we consider results like Holte's (1993). Holte showed that degenerate (one-level) decision trees, called "decision stumps." performed well in terms of accuracy for many commonly used databases. While the algorithm for learning decision stumps is very fast, the method prohibits the learning of complex concept descriptions. Nonetheless, the decision-stump results suggest that a fast, but simple, learning algorithm may ho an effective tool for scaling up to very large databases. Catlett (1991a; 1991c) applied the strategy of simplifying a learner's representation language to the problem of scaling up, and showed that the discretization of numeric attributes can reduce the run time of a decicion-tres karnor aften without a corresponding decrease in accuracy.

A sccond strategy is to optimize a learning program's search and representation as much as possible. Optimization may involve the identification of conctraints that can be exploited to reduce algorithm complexity, or the use of more efficient data structures (e.g., bit vectors, hash tables, binary search trees). Segal and Etzioni's BruteDL (1994) is a highly optimized rule learner, which uscs clever search-reduction terhnirues as well as efficient data structures. When learning time is an issue, such code optimization is good engineering practice and complements the other methods of scaling up that we describe below. However. when fast simple methods are not adedrate and optimization is not enough, other strategies are necessary to scale up learning methods.

The most common method for coping with the infeasibility of learning from very large data sets is to select a smalter sample from the initial data set Catlett (1991a) studied a variety of strategies for sampling from a large data set. Despite the advantages of certain sampling strategies, Catlett concluded that they are not a solution to the problem of scaling up to very large data sers Samping does not aderuately addrose either of the two main reasons for using large data sets; small samples generally reduce accuracy and inhibit learning infrequent special cases.

Catlett (1991a; 1997) also sudied srategies for reducing the complexity associated with description languages containing numeric attributes. He found that by looking at subsets of examples when scarching for good split values for numeric attributes, the run time of decisinn-tree learners can be significantly reduced, without a corresponding loss in accuracy. Even with these strategies in place, the run time of the learners is still fincar in the number of examples, so learning with very large data sets can still be prohibitively expensive. These techniques are complementary to the methods described below lor learning in parallel.

Incremental batch learning (Clearwater, Cheng, Hirsch \& Buchanan, 1989) is a cross betxxeen sampling and incremental learning (Schlimmer \& Fishor, 1086; Utgoff, 1089). 
Incremental batch learners process subsamples of examples in sequence to learn from large training sets. Incremental batch learning has been used to scale up to example seis that are too large for pure batch precessing (Provost \& Buchanan, 1995). Such an approach is effective becausc even for learners that, in principle, scale up linearly in the number of examples, if the entire example set does not fit in main memory, memorymanagement thrashing can render the learner uscless.

Still another approach to scaling up has been sludied by Gaines (1989), though Gaines' primary goal was to unify manual and automatic knowledge acquisition. In particular, Gaincs analyzed the extent that prior knowledge reduces the amount ol data needed for effective learning. Unfortunately, pinpointing a small set of relevant domain knowledge begs the very question of machine learning. Therefore techniques for using background knowledge must scale up to large knowledge bases. Aronis and Provost (1994) use parallelism to enable the use of massive networks of domain knowledge to aid in constructing new terms for inductive learning.

We now discuss an important class of strategies that deal with very large problems by decomposing the learning problem and using parallel machines to process the different pieces simultaneously. Three approaches to parallelization can be identified. First, in the coarse-grained approach, the data are divided among a set of processors; each processor (in parallel) learns a concept description from its set of examples, and the concept descriptions are combined. Shaw and Sikora (1990) lake this approach using a genetic algorithm to combine the multiple concept descriptions, but do not experiment with very large data sets. Chan and Stolfo $(1993 a ; 1993 b)$ also take a coarse-grained approach and allow different learning programs to run on different processors. Their approach takes advantage of existing learning algorithms-only the parallel infrastructure needs to be programmed. Not unexpectedly, as with sampling, such techniques may degrade classification accuracy compared to learning with the entire data set. Provost and Hennessy (1994) also use a coarse grained parallelization, where the individual learners cooperate such that it is guaranteed that cach rule is considered acceptable to the distributed learner if and only if it would be considered acceptable to a monolithic leamer using the entire data set. This approach has been successful with very large data sets. Coarse-grained parallel learning algorithms utilize loosely coupled computers in a distributed processing setting. and could also be implemented successfully on a MIMD (multiple instruction multiple data) parallel architecture.

In the sccond approach to parallel learning, rule space parallelization, the search of the rule space is decomposed such that different prosesors search different portions of the rule space in parallel. This type of decomposition is similar to that used in parallelizing other forms of heuristic search. Although some have stated that massively parallel SIMD (single instruction multiple data) machines are inherently unsuitahlo for parallel heuristic search (Bobrow, 1993), several researchers have implemented heuristic search routines (IDA*) on SIMD architectures with impressive results (Cook \& Lyons, 1993; Powley, Ferguson \& Korf, 1993; Mahanti \& Daniels, 1993). In this work, portions of the search tree are given to the processors, each of which performs a heuristic search. Previous work has also dealt with search on MIMD machines; lor example, Rao and Kumar discuss pas allet depth-first scarch (Kumar \& Rao, 1987; Rao \& Kumar 1987). 
In this mold, Cook and Holder (1990) used the CM-2 Connection Machine for a rule-space parallelization of AQ (Michalski, Mozetic, Hong and Lavrac, 1986). AQ was parallelized by specializing all elements of a star (i.e., an overly general concept description) simultaneously instead of using a beam search. In their approach, $2^{15}(32 \mathrm{~K})$ processors on the CM-2 can handle problems with fifteen features or fewer. However, a tuaxintun of fiften features imposes a sirict limitation on the utility of a lcarnung program. In general, this type of parallelization does not address the problem of very large data sets. If rules are distributed across processors, each processor will cither have to deal with all the data (which does not address the inability of current processors to deal with massive data sets) or cach processor will have to deal with subsets of the data (which would run into the same problems as subsampling). Also, Ioad balancing becomes an issue in order to take full advantage of the parallel processing power. Load balancing and interprocess communication add additional overhead. Cook and Holder (1990) also discuss (without implementing) a rule-space paraliclization of ID3 (Quinlan, 1986), but conclude that it is "actually very difficult to implement" and does not provide "much benefit over the sequential ID3 procedure." Cook and Holder (1990) take a similar approach to parallelizing the perceptron method, and there has been work using rule-space parailetism to scale up other connectionist methods (Kumelhart, Hinton \& Williams, 1986); Ior example, Zhang, Mckenna, Mesirox and Waltz. (1989) utilized the massive parallelism of the CM-2 in a parallelization of a backpropagation neural network.

The third parallelization approach stems from the identification of the major bottleneck in learning from very large data sets and the distribution of the computation that addresses that bottleneck. More specifically, many inductive learning programs fall under the generate-and-test paradigm. In typical artificial intelligence search problems, the major computational cost is due to the fact that many nodes are generated. Thus, previous work on using massively parallel search has concentrated on distributing both the generation and testing of nodes across many processors. However, search for inductive learning differs from most other AI searches --in inductive learning the cost of evaluating a node is very expensive. Nodes in the search tree (e.g., partial rules or decision tree branches) are hypothesized and each must be matched against many examples. The results of this match guide the generation of subsequent hypotheses. For a problem with more than a few hundred examples, this matching dominates the computation.

Thus, our approach utilizes parallel matching. Our approach is similar to that taken by Lathrop's ARIEL system (Lathrop, Webster. Smith \& Winston, 1990); the example set is distributed to the processors of a massively parallel machine. ARITI, was not run on data sets larger than a few hundred examples, however, for two reasons. First, the biological problem being investigated only consisted of several hundred examples, and second. using ARIEL's method of decomposition at few hundred insiances was the maximum possible on the available $8 \mathrm{~K}$-processor CM-2 Connection Machine (Lathrop, 1995).

Stanfill and Waltz (1988) use a parallel matching approach for casc-based learning from very large databases. Their Memory-Based Learning (MBL) approach uses a Connection Machine to find the most similar instance from a very large database. This approach it: inhorently different from parallelixing the type of genoralization algorithom aducssed 
in our work. In MBL all processing is done when a new example is classified, but in our approach learning a concept description precedes classification with that concept description; a parallel machine is not necessary for classitication. A parallel MBL-like approach would only be suitable for the batch classification of a large set of examples, due to the overhead of loading the data onto the parallel machine. Furthermore, MBLstyle learning does not make interesting special cases apparent, because it does not form explicit generalizations.

\section{The RL Learning Program: Sequential and Parallel Variations.}

The RL loarning program (Provost, Buchanan, Cloarwater \& Lee, 1093; Clearwater \& Provost, 1990) is a descendant of Meta-DENDRAL. (Buchanan \& Mitchell, 1978). RL uses a heuristic search algorithm to generate a series of if-then rules and lests each of them against a set of data. In practice, RL is often used to find interesting individual rules. However, the set of rules learned by RL forms a disjunctive class description, which can be optimized with standard techniques as described, for example, by Quinlan (1987).

RI, performs a straightforward, general-to-specific search of the space of rules defined by conjunctions of attribute-valuc pairs (features). The goal of RL's scarch is to find rules that eatisfy user defined criteria. In particular, in the experiments below RL searches for rules that satisfy two thresholds: the positive threshold, which specifies the minimum number of positive examples a rule must cover, and the negative threshold, which specifies the maximum number of negative examples a rule may cover. The use of thresholds relaxes constraints on the coverage of discovered rules, mitigating the effects of noise in the data and/or the effects of an inadequate represcntation language.

Each rule has a set of conditions and a predicted class, which RI cvaluates statistically. The space of possible rules includes all possible combinations of conditions, so the size of the search space grows exponentially with the allowable number of conditions in a rule. RL. uses a beam search to ensure that the time complexity of the search is linear in the number of conditions. The beam evaluation function is defined by the user; for the experiments reported here we used a signal-to-noise function that is, roughly, the percontage of positive examples covered by a rule divided by the percentage of negative examples covered. Fach rule is tested against the entire set of data to calculate performance statistics. This introduces only a linear factor into the complexity of the algorithm when the data are described solely by nominal attributes (an ${ }_{2} l o g h$ factor if the program searches for numeric features). Nonetheless, for data sets with more than a few hundred examples, the testing dominates the computation. For data secs with millions of examples the time spent checking rules against data can run into days or weeks, making learning from very large data sets impossible from a practical standpoint.

SIMD paraltel architectures, such as the CM-2 Connection Machine, consist of a frontend workstation that issues instructions to thousands of processors to be cxceuted simultaneously. This provides a perfect match to generate-and test inductive learning programs such as RI. The front-end generates partial ruies, and each partial rule is tested on data esiding on individual processurs. As cacti sule is created it is broadeast to all the CM-2 
processors which simultaneously match it against the data residing on that processor. The results of these matches are sent back to the front-end to guide the generation of subsequent rulcs.'

On a sequential machine checking a rule against $n$ data items takes cn time, but on a SIMD machine with $p$ processors, it takes only $c^{\prime} n / p$ time to check a rule. Since $p$ is large lor massively parallel machines (e.g., up $1004 K$ on a CMT-2), this is a very favorable speedup despite the fact that on the CM-2 individual processors are relatively slow, bit-serial processors (i.e., $c^{\prime}$ is large).

Notice that processors check a rule against only the data items stored in that processor's local memory. There is no communication overhead because there is no interprocessor communication. In theory, collecting results from individual processors and relurning the aggregale to the front-end takes $\log n$ time, but special hardware makes this tactor insignificant.

This complexity analysis may obscure the main point, which is that parallelism allows us to scale up to data sets several orders of magnitude larger than previously possible. Since checking $k$ rules takes time proportional to $k n / p$, a large $p$ allows us to increase the number of data items, $n$, thereby making it possible to learn more accurate concept descriptions, and to learn small disjuncts that previously were not practically possible to learn.

\section{Experimental Results.}

This section describes the results of running scquential and parallel versions of RL on synthetic and real-world data sets of a million or more examples. We used synthetic data to better control experimental parameters, and real-world data to illustrate that the scaling cnubled by massive parallelism does indecd lead to uscful, novel discoverics. In the results presented below, sequential RL. (a relatively fast C-language version of the program) was run on a dedicated DECstation 5000 with $32 \mathrm{M}$ main memory. Parallel RL was run on a CM-2 Connection Machine with $8 K$ processors.

\section{I. Fxperiments with Synthetir Dath}

We designed a learning task that had a concept description with disjuncts of various sizes. There were a total of one million examples, and the concept to be learned included 0.5 million positive examples. As Table 1 indicates, each example of the concept consisted of 27 features: 7 significant features, and 20 with random values. For the (positive) examples of the concept, one example had a "I" for the first significant feature, and "0" for the rest of the significant features; 10 examples had a "0" for the first significant feature, "l "for the second, and " 0 " for the rest of the signiticant features; . ; 100,000 examples had a "()" for the first five significant features, "l" for the sixth, and "0" for the rest of the significant features; the remainder of the 0.5 million examples of the concept had " 0 " for the first six significant features, and a "1" for the seventh significant feature. The 0.5 million examples of the complement simply had " 0 " for cach significant foature. 
All examples of the concept and its complement had random values for the remaining 20 features.

Table 1. Design of the Synthetic Concept.

\begin{tabular}{|c|c|c|c|c|c|c|c|c|c|}
\hline \multicolumn{10}{|c|}{ Positive Examples } \\
\hline 1 Example: & 1 & 0 & 0 & 0 & 0 & 0 & 0 & + & 20 random digits \\
\hline 10 Examples: & 0 & 1 & 0 & 0 & 0 & 0 & 0 & + & 20 random digits \\
\hline 100 Examples: & 0 & 0 & 1 & 0 & 0 & 0 & 0 & t & 20 random digits \\
\hline 1000 Examples: & 0 & 0 & 0 & 1 & 0 & 0 & 0 & + & 20 random digits \\
\hline 10000 Examples: & 0 & 0 & 0 & 0 & 1 & 0 & 0 & + & 20 randorn digits \\
\hline 100000 Examples: & 0 & 0 & 0 & 0 & 0 & 1 & 0 & + & 20 random digits \\
\hline Remainder of $0.5 \mathrm{M}$ Examples: & 0 & 0 & 0 & 0 & 0 & 0 & 1 & + & 20 random digits \\
\hline \multicolumn{10}{|c|}{ Negative Examples } \\
\hline 0.5M Examples: & 0 & 0 & 0 & 0 & 0 & 0 & 0 & + & 20 randem digirs \\
\hline
\end{tabular}

RL allows the user to specify thresholds of acceptability. Typically, a user specifies that an acceptable rule must cover a substantial portion of the positive cxamples, while allowing it to cover some small number of negative examples. For this experiment we specified that a rule can cover as few as one positive example, but must not cover any negative examples. Furthermore, to eliminate extraneous search from the comparison of run times we specified that RL was to learn only rules with a single conjunct. Thus, to characterize the concept RI, was forced to learn a rule for each parl-with its single conjunct specilying a "l" in one of the significant features. The disjunction of these rules covered the concept. This test was designed to sec if sequential and parallel RI. could in fact learn rules of various sizes from a large set of data, and to compare the run times.

Figure 1 shows the time required by both sequential RL and parallel RL to learn rules that characterize the concept. The sequential version was run on data sets up to $70 \mathrm{~K}$ examples, at which point it became infeasible to run it on larger data sets. We project that it would have taken more than 20 hours to run sequential RI, on all the data. Parallel RL took less than one minute to learn rules on all data sets up to one million examples.

A close examination of the graph in Figure l reveals that the limes for parallel learning form a step function. Figure 2 shows the parallel learning times in detail. Without the sequential times in the graph to swamp the much smaller parallel times, the step function is more apparent. The CM-2 was run with $8 \mathrm{~K}$ actual processors. For data sets with more than $8 \mathrm{~K}$ cxamples virtual processors had to be allocated. That is, cach proessor cmulated several virtual processors (and, therefore, stored multiple data items). Each time new virtual processors had to be used to accomodate a larger data set the overall computation time reflected the increased cost of cmulation. Virtual processors must be allocated in powers of 2 , so as to emulate an entire hypercube. Thus, the bottom of the steps visible in the graph shown in Figure 2 correspond to example sets of size $64 \mathrm{~K}$, $128 \mathrm{~K}, 256 \mathrm{~K}$, and $512 \mathrm{~K}$. One cxtra example pusties the run time up to the next step. 


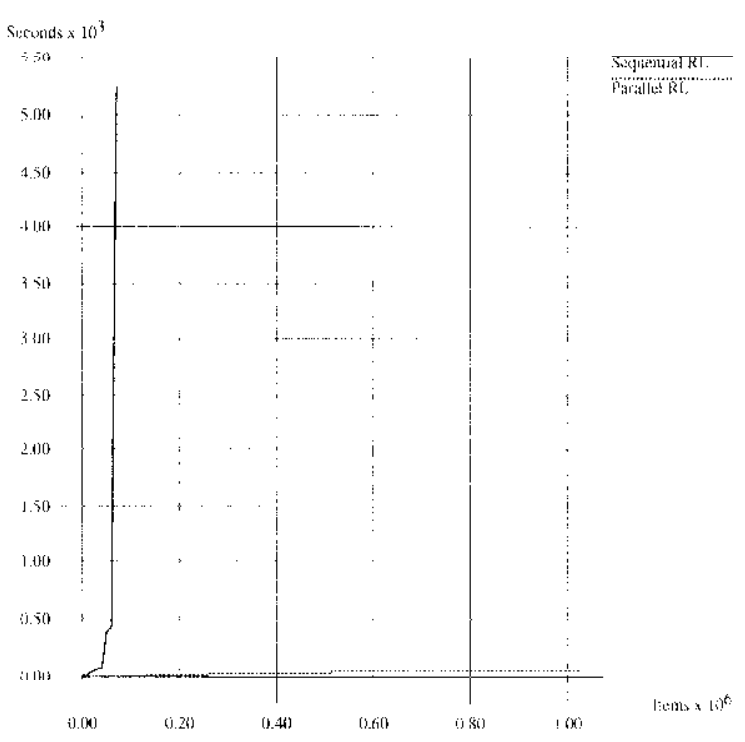

Figure 1. Time Requured to Learn on Synthetic Data.

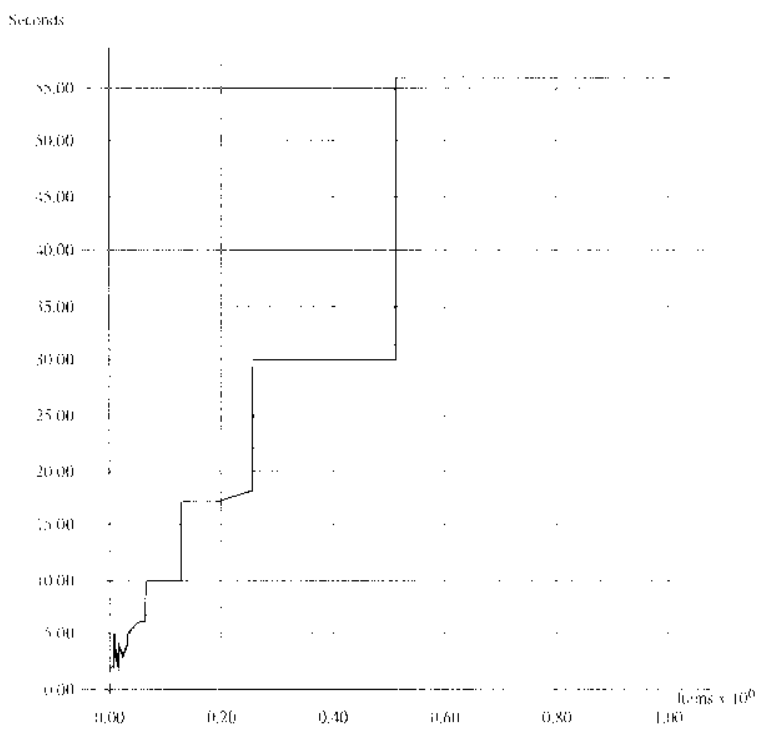

Figute 2. Timb Required to Learn on Synthetic Data-Detail for Parallel RI. 
Two observations are relevant here. First, the basic parallel operation-evaluating a predicate across independent data items distributed across many processors---does not involve interprocessor communication. Sccond, cach processor of the CM-2 is a relatively weak bit-serial processor, so the speedup factor is not nearly $8 K$, but the use of thousands of such processors produces an overall dramatic effect. Subsequent architectures that combine large numbers of more powcrful processors (such as the CM-5 or Cray's T3D) will give more impressive speedups.

Parallelizing RL, allowed it to learn rules that practically are impossible for the sequential program to learn on current workstations. On the test workstation, the maximum practical sample size for the sequential RL is $50 \mathrm{~K}-100 \mathrm{~K}$ examples, but the chance is nearly zero that such a sample will contain an adequate representation of all parts of a concept that is made up of very small disjumets. Fenember that it is often thexe special cases that particularly interest scientists. In contrast, parallel RL learned all rules necessary to cover the positive examples with a million training items.

\subsection{Experiments on a Public-Health Database.}

We analyzed a data set comprising U.S. Department of Health birth records linked with records of infant deaths. Parallel RL was used to learn rules to predict infant mortality and survival. The database contained 3.5 million records with about 20 fields each. including race, birthweight, and place of birth. This is an example of a problem where the goal is not to form a classifier, since we are not predicting whether new infants are going to survive, but to identify interesting subgroups of the population. Identifying subgroups with unusually high and unusually low infant mortality rates directs further research. The long-term goal of such work is to fornulate policies that will reduce the nation's infant mortality rate. and the rate for particular subgrouns.

Figure 3 shows the learning times required to learn with this data set, which are similar to those obtained with the synthetic data. Notice, in particular, that the sequential prograni becomes practically useless at approximately the same number of training examples as it did with the synthetic data.

The massively parallel system learned the rule, known by experts in the field, that African-Americans have a high rate of infant mortality $(1.88 \% \mathrm{vs} .1 .10 \%$ for the general population). It also learned the small rule that Japanese- $\Lambda$ mericans have a low rate of infant mortality $(0.79 \%$ ), and the even smaller rule that Japanese- $\Lambda$ mericans living in Fast Coast states have a very low rate of infant mortality (0) $18 \%)^{2}$ it is importan to remember that the dataset contained approximatcly 3.5 million records; so the small difterences we see here are significant.

In addition, the analysis of the infant mortality datahase with parallel RI uncovered at surprising relationship that has led to considerable follow-up research with our public-. health collaborators. Public-health researchers are concerned about the disparity in infant mortality rates between African-Americans and the general population. In the general population earlier prenatal care correlates with a reduction in infant mortality rates: however, Rl discovered that for African-Americans, earlier prenatal care is correlated with higher infant mortality rates. Statistical tests show the relationship to be significant 


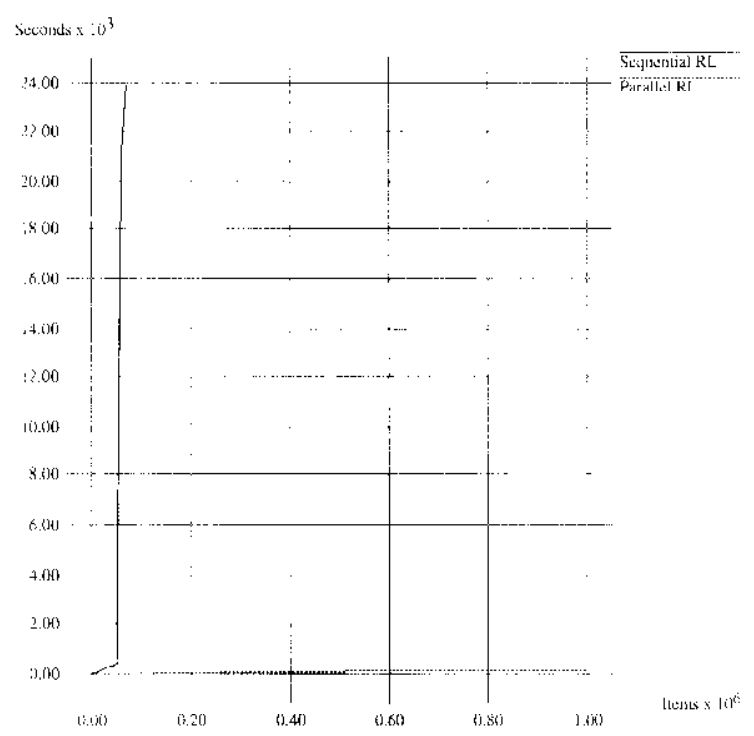

Figure 3. Time Required to Learn on Health Department Data

even after controlling for confounding variables. Further analysis has explained the relationship only partially. These results are currently being written up for submission to a public-health journal (Sharma, Provost, Aronis, Mattison \& Buchanan, 1994).

\section{Conclusions}

Massively parallel matching succeds hecause it atlacks a specific botileneck encountered with very large problems, i.e., matching hypotheses against huge data sets is computa. tionally expensive. As opposed to previous work (Cook \& Holder. 1990), we did not attempt to parallelize the entire algorithm. The generation of hypotheses takes place on the supercomputer's frontend workstation. This makes sense since the results of matching hypotheses against the data guide the generation of subsequent hypotheses (and so there is a serial nature to this portion of the algorithm). Each hypothesis is independently checked against each data item, which ean be done in parallel. However the sequential bottleneck is not avoided entirely with the CM-2. I oading the data into the CM-2 processors is a sequential task that can take several minutes for one million data points. Coupling this overhead with the performance numbers depicted in Figures 2 and 4, we conclude that the massively parallel version is preferable when the number of examples is greater than $10 \mathrm{~K}$. This conclusion is based on domains with approximately 20 attributes, but it should hold for domains with more or fewer attributes, up to the point where the memory of the individual processors is exhausted. 
Parallel matching applies to generate-and-test learning programs in general, such as MetaDENDRAI_-style rule learners akin to RL; parallel matching would undoubtedly enable dramatic scaling for systems such as BruteDL, (Segal \& Etrioni, 1994), where efficiency is a primary concern. Parallelizing some other generate-and-test machine learning programs would be slightly less straightforward, but we believe our basic approach would succeed. For example, consider a version of Mo3 that exploits parallel matching. Roughly speaking, as decision-tree partial paths were generated by the front end, they would be matched against all of the data in parallel. This procedure does not exploit the recursive partitioning nature of sequential ID3, which matches partial paths against increasingly smaller subsets of the data. Thus, speedups would not be as large as for RI, because often many processors would be "extraneous" when matching a decision-tree partial path. Nonetheless, we stull would expect specdups to be dramatic lor very large example sets.

For other lcarning methods where concept description generation and testing are too closely coupled, parallel matching will be either awkward or impossible. $\wedge$ s an extreme example, it would be difficult to use this method to parallelize backpropagation learning for a neural network.

In summary, for some learning tasks, like the exploratory analysis of the infant mortality data, learning small rules is very important. In order to learn small rules, it is necessary to have very large samples so that the algorithm will see cnough cases to form a rule (with confidence). However, learning with sample sets containing one million or more examples is infeasible on standard sequential machines. We have shown that massive parallelism is an effective way to scale up inductive leaming to large data-analysis problems

\section{Acknowledgements}

We thank our public health collaborators, in particular, Ravi Sharma and Don Mattison, for their excitement about using machine learning for scientific data analysis and for giving us a reason for turning our musings about scaling up learning prograrns into reality. Wo also thank Bruce Buchanan for his eternal confidence that machine learning can make a difference on real-world discovery problems. Doug Fisher and our anonymous referees provided helpful content and editorial comments, and Jason Catlett. Philip Chan, Chris Matheus, Andreas Muelier, and Gregory Piatetsky-Shapiro provided comments on the reasons for and methods for scaling up to very large data sets. Finally, we gratefully acknowledge the support of the Pittsburgh Supercomputing Center, the W.M. Keck Center for Conputational Biology, and NYNEX Science and Technology, Inc. 


\section{Notes}

1. Stuliv and Shaw designed DADO a parallel tree-structured machine for production system matchng. to deal with large production systems (Stolfo \& Shaw, 1982; Stolto, 1987). DADO was based on the principle that in production systems, the matching of each rule against working memory is independent of the others. In principle, our approach to parallel leaming would work well on DADO; however, our representation is much simpler, so many of ISADO's capabilities would be wasted.

2. The feature tas coast does not appear in the original data set. It was created by a constructive induction program described by Aronis and Provost (1994).

\section{References}

Aronis, J.M., \& Provost, F.J. (1994). Efficiently constructing relational features from background knowledge: for inductive machine learning. Working Notes of the AAAl-94 Workshop on Knowledge Discovery in Databases (pp. 347-358). Scaltle WA: AAAI.

Bobrow, D. (1993). Editorial introduclion. Artificial Intelligence, 60, 197.

Buchanan, B., \& Mitchell, T. (1978). Model-directed learning of production rules. In D. Waterman \& $\mathrm{F}^{\mathrm{H}}$ Hayes-Roth (Eds.), Patern Directed Inference Systems. New York, NY: Academic Press.

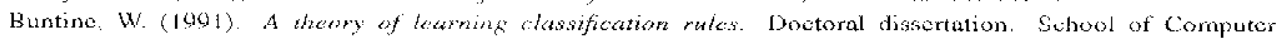
Science, Universiry of Technology. Syciney. Australia

Catlett, J. (1991a). Mexainduction: mathine learning on very large databases. Docloral dissertation. Bassicr Department of Computer Science. Unversity of Sydney, Australia.

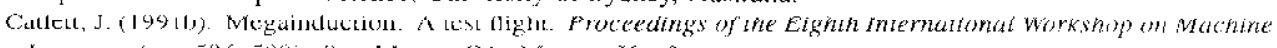
learning (pp. 596 .509)). San Marco, (.A: Morgan Kaufmann

Catlett, J. (1991c). On changing continuous attributes into ordered discrete attributes. Proceedings of the European Working Sestion on learning (pp. 164.178). New York, NY: Springer-Verlag.

Catlett, J. (1992). Peepholing: choosing attributes efficiently for megainduction. Proreedings of the Ninth hiernational Conferente on Macfine fearning (pp. 49-54). San Matco, ( A: Morgan Kaulinam.

Chan, P. \& Siolto, S. (1993a). Meta-learning for multistrategy and parallel learning. Protedings of the Second International Workshop on Multistrategy Learning (pp. 150-165). Fairfax. VA: Center for AJ. George Masion University.

Chan, P. \& Stolfo, S. (1993b). Toward paraltel and distributed learning by meta-learning. Working Noles of the AAAl-9.3 Workshop on Knowledge Discovery in Databases (pp. 227 240). Seattle, WA: ANA1.

Clearwater SH. Chene. TP Firsch. II \& Buchanan, R G (1989) Incremental hatrh liarning pracesedings of the Sixth Internationat Workshop on Machine Learning (pp. 366-370). San Mateo, CA: Morgan Kaufmann.

Cleanwater, S. \& Provost. F. (1990). R1 A: A tool tor knowledge-hased induction. Proceedings of the Second International IIFIE Cimference on Toolis for Artificial Intligence (pp. 24 30). Los Namitos. CA: IFEF Computer Sociexy Prosit

Cook. D., \& Holder, L. (1990). Accelerated harning on the connection machine Proceedings of the Sesond IEEE Symposium on Parallet and Disuibuted Processing (pp. 448-454). 1.os Alarnitos, CA: IFEE Computer Society.

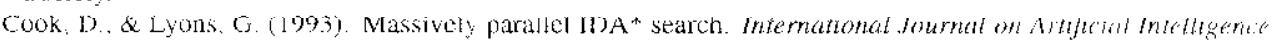
Tools. $2,16.3180$

Danyluk, A.P., \& Provost, F.J. (1993). Sinall disjuncts in action: 1.tarning to diagnose errors in the telephone network local loop. Procedings of the Tenth International Conference on Marhine lectring (pp. 81 88) San Mateo. CA: Morgan Kaufrmann.

Ganes. B.R. (1989). An ounce of knowledge is worth a ton of data: Quantitative studies of the rade-oll between expertise and data based on statistically well-founded empirical induction. Procedirts of the Sixh

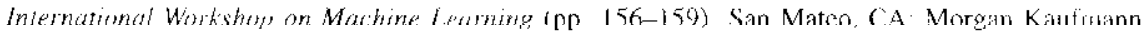

Gordon, D), \& desfurdins, M. (Eds.) (1495). Special issue on bias evaluation and selection. Machine loarmms. 20

Holte, R.C. (1993). Very simple classification rules perform well on most commonly used datasets. Marhne towatrits. $t 1,63$ i $>0$ 
Holte, R.C., Acker, L.E., \& Porter, B.W. (1989). Concept learning and the problem of small disjuncts. Proceedings of the Eleventh International Joint Conference on Artificial Intelligence (pp. 813 818). San Mateo, CA: Morgan Kaufmann.

Kumar, V., \& Rao, V. (1987). Parallel depth-first search, Part II: analysis. Internationat Journal of Parallel Programming, 16, 501-519.

Lathrop, R.H. Webster, T.A., Smith, T.F, \& Winston, P.H. (1990). ARIEL: A massively parallel symbolic leaming assistant for protein structure/function. In P. H. Winston \& S. Shellard (Eds.), AI at MTT: Enzanding Frontiers. Cambridge, MA: MIT Press.

Lathrop, R.YI. (19)5). Massachusetts Institute of Technology. Personal Communication.

Mahanti, A \& Daniels, (. (1993). A SIMD approach to parallel heuristic search. Artificial tritelligence. 60, 243282

Michalski, R., Mozetic, I., Hong, J., \&. I avrac, N. (1986). The Multi-purpose incremental lcarning system $\mathrm{AQ} 15$ and ts testing application to three medical domains. Proceedings of the Fith Narional conference on Artificial Intellicence (pp. 1041-1045). Menlo Park, CA: AAAJ-Press.

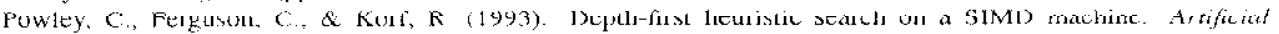
Intelligence: $60,199 \cdots 242$.

Provost, F.\}., \& Buchanan, B.G. (1995). Inductive policy: The pragmatics of bias selection. Machine Learning, $20,35 \cdots 61$

Provost, FJ Buchanan, BG. Clearwater, S.H, \& Lee, Y. (19y.5). Machine learming the servet of ex ploratory sctente and thyineering: A case study of the RL induction program. Technical Report 1SL-93-6 Intelligent Systems Laboratory, Computer Science Department, University of Pittsburgh, Pittsburgh, PA

Provost. F.J., \& Hennessy, D) (1994). Distributed machine learning: Scaling up with coarse-grained parallelism. Procedings of the Second Internatonal Conference on Intelligent Systerts for Molecular Biology (pp. 340347). Menlo Park, CA: AAAl Press.

Quinlan, J. (1986). Induction of Decision Trees. Machine Learning, 1, 81-106.

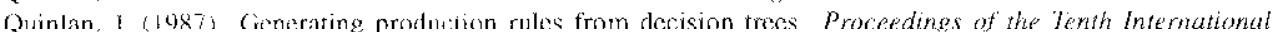
Joint Conferente un Artificial Intellisence (pp. 304--307). San Mateo, CA: Morgan Kaufmann.

Rao, V. \& Kuniar V. (1987). Parallel depth-first search, Part I: Implementation. Inresnationaf Journal of Parallet Programiming, 16, 479-499

Rumelhart, 1).E., Hincon, G.F. \& Williams, R.J. (1986). Learning imternal representation by error presagation In D.E. Runclhart \& J.L. MCClelland (Fds.), Parallel Distributed Processing. Cambridge MA: MI'T Press

Schlimmer I.C. \& Fisher. 1). (1986). A case study of incremental concepe induction. Prockedings of the tifh National Conference on Articiul Inteltigence (pp. 496 501). San Mateo. CA: Morgan Kaufnamn.

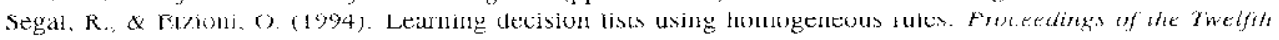
Nationat c'onferesce on Artificial Inielligence (pp. 619 625). Menlo Park, CA: AAAl Press.

Shatma, R. Provust, F., Aronis, 3., Matison, D., \& Buchanan, B. (1995). An whe ppected relationship between the timing of entry into prentat care race, and infant morality. In preparation. Iniversity of Pidtsburgh. P'ittsburgh. PA

Shaw, M. J. \& Sikon. R. (1990). A distributed problem-solving approach oo inductio teraning. lechnical Report CMU RI TR 90 26, Robotics Institute, Carnegie Mellon Universily, Pirtsburgh, PA.

Stanfill. C.. \& Wilu 1) (1988). The memory-based reasoning patadigm Proicedings of a Workshop on Case-Based Re'twom (pp. 414 424). San Mateo. CA: Morgan Kaufunann.

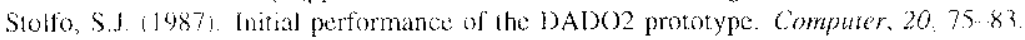

Stolfo, S.J. \& Shaw, DF (1982). DADO: a tree structured machine architecture for production systems.

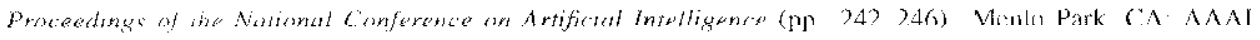
Press.

Cigofi, PE. 1989, Incremental induction of decision trees. Mathine Learming, + $161 \ldots+186$.

Weiss. (i.M. (1905), Learmeng with Small Disjuntes. Technical Report MI-TR-39, Department ol Compuler Stience, Kutgeas binvasity, View Butuswick. NJ

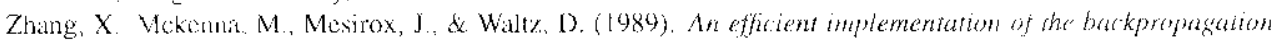
agorihm on the conrection mathine (M-2. Technical Report RL89-1, Boston, MA. Thinking Machines Corporation

Recerved June 30. 1994

Accepted July 30 1945

Final Manuscript $\mathrm{I}_{\mathrm{t}} \mathrm{ly} 10,1995$ 